\title{
COMPORTAMENTO E PARÂMETROS FISIOLÓGICOS DE LEITÕES NAS PRIMEIRAS 24 HORAS DE VIDA
}

\author{
Valuation of behavior and physiologic parameters of the first 24 hours of sucking pigs life
}

\author{
Rony Antonio Ferreira ${ }^{1}$, Julien Chiquieri ${ }^{2}$, Pedro Pierro Mendonça ${ }^{2}$, Thiago Vasconcelos Melo $^{2}$, \\ Mariana Duran Cordeiro ${ }^{3}$, Rita da Trindade Ribeiro Nobre Soares ${ }^{4}$
}

\begin{abstract}
RESUMO
Um experimento foi conduzido no setor de Suinocultura da Universidade Estadual do Norte Fluminense, com o objetivo de avaliar o comportamento e parâmetros fisiológicos de leitões nas primeiras 24 horas de vida. Foram utilizados 82 leitões de genética comercial para crescimento rápido, sendo realizadas observações a cada cinco minutos do comportamento dos animais. Foram mensuradas as temperaturas retal e de superfície, além da freqüência respiratória em intervalo de uma hora. Os resultados obtidos com o estudo comportamental demonstraram que os leitões passam mais da metade $(53,8 \%)$ do seu primeiro dia de vida dormindo dedicando somente $31,2 \%$ à ingestão de leite. A temperatura retal dos leitões recém nascidos e a temperatura superficial da pele aumentaram $(\mathrm{P}<0,05)$ após nove horas de vida. Entretanto, a temperatura superficial no pernil não foi influenciada $(\mathrm{P}>0,05)$ nas primeiras 24 horas de vida. A freqüência respiratória não foi influenciada $(\mathrm{P}>0,05)$. Leitões recém-nascidos com genética para alta deposição de tecido magro apresentam reação ao ambiente logo nas primeiras horas de vida. A elevação das temperaturas retal e superficial dos animais evidenciaram tentativa de controle termorregulatório a partir da nona hora de vida.
\end{abstract}

Termos para indexação: Suínos, temperatura retal, termorregulação.

\section{ABSTRACT}

An experiment was carried out in the swine sector of North Fluminense State University to evaluate the behavior and physiological parameters of piglets in the first 24 hours of life. A total of 82 growing piglets from commercial genetic lines were used. Observations of individual piglet behavior were made each five minutes. Rectal and superficial temperature of the piglets were evaluated. Moreover, respiratory frequency was observed each one-hour interval. Results showed that piglets take a little more than half of their first day of life time sleeping $(53,8 \%)$ and only $31,2 \%$ sucking. Rectal and superficial temperature increased after nine hours of life. However, superficial temperature of ham and respiratory frequency were not influenced. Newborn piglets from commercial genetic lines showed reaction to environment in the first hour of life. Moreover, the increase in rectal and superficial temperature evidenced attempt to maintain thermoregulatory control after nine hours of life.

Index terms: Swine, rectal temperature, thermoregulation.

\section{(Recebido em 21 de setembro de 2006 e aprovado em 26 de abril de 2007)}

\section{INTRODUÇÃo}

O leitão recém-nascido apesar de ser neurologicamente desenvolvido, ainda é fisiologicamente imaturo e algumas mudanças importantes ocorrem no início de sua vida, habilitando-o para a sobrevivência. Estas mudanças ocorrem principalmente nas primeiras semanas de vida, onde seu sistema termoregulatório ainda é ineficiente em manter eficazmente sua homeotermia.

Atualmente, as modernas linhagens genéticas de suínos apresentam desempenho produtivo diferenciado, em comparação àquelas disponíveis há duas décadas. Brown-Brandl et al. (2001) evidenciaram diferentes padrões termorregulatórios para suínos em fases de crescimento e terminação. Entretanto, poucos relatos na literatura destacam o comportamento e fisiologia de leitões lactentes. A maior parte dos estudos envolvendo comportamento é realizada com suínos em crescimento e terminação (SCOTT et al., 2006). Dados não publicados reportaram que os leitões realizam, em média, 20 mamadas ao dia com duração de 20 a 30 segundos, ingerindo de 20 a $60 \mathrm{~g}$. de leite por mamada

\footnotetext{
'Doutor, Professor Associado - Departamento de Ciências Agrárias/CCA - Universidade do José do Rosário Vellano/UNIFENAS - Rodovia MG 179, Km 0 - Cx. P. 23 - Alfenas, MG - rony.ferreira@unifenas.br

2Zootecnistas, Doutorandos em Produção Animal - Centro de Ciências e Tecnologias Agropecuárias/CCTA - Universidade Estadual do Norte Fluminense Darcy Ribeiro/UENF - Avenida Alberto Lamego, 2000, Califórnia - 28013-600 -Campos dos Goytacazes, RJ - chiquier@uenf.br; ppiero@pop.com.br; thiagovmelo@gmail.com

${ }^{3}$ Doutor, Professora - Centro de Ciências e Tecnologias Agropecuárias/CCTA - Universidade Estadual do Norte Fluminense Darcy Ribeiro/UENF Avenida Alberto Lamego, 2000, Califórnia - 28013-600 - Campos dos Goytacazes, RJ - duran@uenf.br

${ }^{4}$ Professora Associada - Centro de Ciências e Tecnologias Agropecuárias/CCTA - Universidade Estadual do Norte Fluminense Darcy Ribeiro/UENF Avenida Alberto Lamego, 2000, Califórnia - 28013-600 - Campos dos Goytacazes, RJ - rnobre@uenf.br
} 
(LIMA, 1995). Recentemente, Pandorfi (2002) avaliou, mediante observação de comportamento, o fornecimento de diferentes fontes de aquecimento para leitões lactentes, não envolvendo, entretanto, estudos de ingestão de leite ou desenvolvimento termorregulatório.

O leitão recém nascido apresenta limitada reserva energética disponível armazenada na forma de glicogênio e menos de $1 \%$ de gordura no corpo, além de pouco isolamento corporal por pêlos. Dessa forma, torna-se necessário mamar logo após o nascimento, evitando queda drástica nos níveis de glicose do sangue e conseqüentemente da temperatura corporal. $\mathrm{O}$ valor normal de $100 \mathrm{mg}$ de glicose por $100 \mathrm{~mL}$ de sangue do leitão ao nascer pode cair para $10 \mathrm{mg}$ ou menos nos dois primeiros dias caso o animal seja submetido à jejum alimentar. Ele conta, nas primeiras horas de vida, apenas com a glicose catabolizada a partir do glicogênio hepático como sua principal fonte energética, o que é suficiente apenas para cobrir suas necessidades por 15 a 20 horas.

Mesmo mamando, o leitão pode ter hipoglicemia quando associada ao estresse gerado pelo frio ou ainda combinada ao inadequado suprimento de leite pela porca. English (1998) enfatizou que a hipotermia é uma das principais causas de mortalidade de leitões recém-nascidos.

Pereira \& Passos (1998), trabalhando com leitões recém-nascidos nos quais a variação da temperatura corporal foi monitorada, concluíram que o controle da temperatura ambiental com o uso de escamoteadores e aquecimento é imprescindível para auxiliar os leitões recémnascidos na manutenção de sua homeotermia, o que foi confirmado por Pandorfi (2002).

Da mortalidade na maternidade, cerca de $70 \%$, ocorrem na primeira semana de vida. As causas são inúmeras e a grande maioria de natureza não infecciosa, como esmagamento e inanição. A inanição, por sua vez, causada por causa da agalaxia, exposição ao frio ou sangramento do umbigo. Na falta de aquecimento artificial, os leitões que não são amamentados, principalmente, tornam-se hipoglicêmicos e procuram aquecimento junto à mãe. Isso, muitas vezes, resulta no esmagamento destes indivíduos. Os leitões mais fracos são os mais atingidos, representando cerca de $65 \%$ do total de perdas nesta fase.

O melhor ganho de peso na fase de aleitamento apresentará reflexo positivo nas fases subseqüentes de crescimento dos animais. Assim, a descrição do comportamento e de parâmetros fisiológicos de leitões em lactação é de fundamental importância para propor técnicas de manejo que melhor se ajustem às novas linhagens genéticas de suínos existentes nas criações comerciais.

\section{MATERIAL E MÉTODOS}

O experimento foi conduzido no Setor de Suinocultura da Universidade Estadual do Norte Fluminense Darcy Ribeiro (UENF), situado no município de Campos dos Goytacazes (RJ).

As fêmeas suínas durante a fase de gestação permaneceram em sistema de criação coletivo sendo posteriormente transferidas para baias individuais sem o uso de celas parideiras de maternidade, atendendo às recomendações da Comunidade Européia. O galpão de maternidade possui uma área coberta com telha de barro e outra área de solário. A área coberta disponibiliza área útil de $4 \mathrm{~m}^{2}$ e o solário $6 \mathrm{~m}^{2}$ por matriz alojada. Cada baia possui, em toda sua extensão, protetores laterais contra esmagamento acidental de leitões pela matriz e ainda escamoteador com fonte de aquecimento para os leitões.

Os dados foram obtidos em 82 leitões oriundos de sete leitegadas iniciando ao nascimento até as primeiras 24 horas de idade. Todos os leitões foram provenientes de fêmeas primíparas cobertas no terceiro cio, conforme manejo adotado para sincronização do parto. Por ocasião do nascimento, todos os leitões foram pesados sendo realizado o procedimento de orientação da primeira mamada e cuidados com os recém nascidos. Foram avaliados o comportamento e alguns parâmetros fisiológicos.

Para caracterizar o comportamento, foi construído um etograma (DEL-CLARO, 2004) e observada a frequiência que todos os leitões estavam dormindo, mamando ou realizando outras atividades. A observação dos animais foi realizada de forma dirigida (FERREIRA, 2005) de modo a não afetar o comportamento natural dos mesmos. Os animais foram monitorados por um período de 24 horas a intervalos de cinco minutos, de modo a caracterizar o perfil nictemeral de comportamento. As leitegadas foram monitoradas ao mesmo tempo por observadores posicionados estrategicamente, cumprindo uma escala previamente estabelecida.

Os parâmetros fisiológicos avaliados foram: freqüência respiratória, temperatura retal e a temperatura superficial da pele (na nuca, paleta e pernil). Os parâmetros fisiológicos foram mensurados em três animais escolhidos aleatoriamente, de modo a não afetar o comportamento natural dos demais da leitegada. A temperatura retal, a temperatura superficial e a frequiência respiratória foram obtidas desde o nascimento a intervalo de uma hora durante as primeiras 24 horas dos leitões. A temperatura retal foi obtida, por meio de um termômetro clínico digital introduzido no reto de cada animal com alarme sonoro para identificação da constância de temperatura. A temperatura 
superficial foi obtida por meio de termômetro digital a laser com foco dirigido a 20 centímetros, em três pontos do corpo, nuca, paleta e pernil. Para obtenção da freqüência respiratória, foram monitorados os movimentos de flanco de cada animal em 15 segundos e multiplicados por quatro, para obtenção do número de movimentos por minuto.

$\mathrm{O}$ ambiente foi monitorado diariamente por meio de dois conjuntos de equipamentos de medição, compostos cada um por termohigrômetro digital, termômetro de globo negro e anemômetro digital. Os dois conjuntos foram localizados à meia altura do corpo dos animais sendo um no interior do escamoteador, para avaliar o ambiente dos leitões, e outro conjunto foi disposto na baia, de modo a avaliar o ambiente da fêmea suína. Os resultados monitorados de ambiente foram utilizados para cálculo do ITGU (Índice de Temperatura de Globo e Umidade), por melhor caracterizar o ambiente animal nos trópicos, como referenciado por Ferreira (2005).

A água e a ração foram fornecidas à vontade para as fêmeas suínas.

As análises estatísticas das variáveis fisiológicas (temperatura retal, frequiência respiratória e temperatura superficial) foram realizadas utilizando-se o programa computacional SISVAR do Departamento de Ciência Exatas da Universidade Federal de Lavras, segundo Ferreira (2000), adotando-se o teste de Scott-Knott ao nível de 5\% de probabilidade. As variáveis de comportamento obtidas foram avaliados através de estatística não paramétrica, construindo-se gráfico de distribuição de freqüência em planilha eletrônica no programa EXCEL for Windows versão 2000 .

\section{RESULTADOS E DISCUSSÃO}

Durante o período experimental a temperatura média interna da sala manteve-se em $28,5 \pm 3,0^{\circ} \mathrm{C}$, com umidade relativa de $63,4 \pm 6,0 \%$ e temperatura de globo negro de $29,8 \pm 2,9^{\circ} \mathrm{C}$. O índice de temperatura de globo e umidade (ITGU) calculado foi de 78,7 $\pm 3,6$. Os resultados observados nos parâmetros ambientais avaliados permitem caracterizar ambiente de estresse por calor para as matrizes. Como referenciado por Ferreira (2005), a faixa ideal de temperatura para a matriz deveria ser de 12 a $15^{\circ} \mathrm{C}$, não devendo ultrapassar a temperatura crítica superior de $27^{\circ} \mathrm{C}$. Matrizes suínas em lactação mantidas sob condições de calor diminuem o consumo de alimento resultando em menor produção de leite, tendo, como conseqüência, comportamento diferenciado dos leitões, que dedicam maior tempo às mamadas, fato que prejudica também as fêmeas, em razão do menor tempo disponível para ingestão de alimento. Este complexo comportamental, na fêmea suína, poderá resultar em maior intervalo desmama-cio fértil, tendo como conseqüência, aumento dos dias não produtivos da matriz na granja.

Na Tabela 1 estão apresentados os resultados dos parâmetros fisiológicos (freqüência respiratória, temperatura retal e temperaturas superficiais da nuca, paleta e pernil). A frequiência respiratória não sofreu alterações significativas $(\mathrm{P}>0,05)$ durante as primeiras horas de vida dos leitões. A alta variabilidade dos resultados observados pode ter influenciado a resposta analisada. Entretanto, existe correlação direta entre a freqüência respiratória e a taxa metabólica do suíno. Este fato pode ser explicado pela relação direta existente entre o aumento da freqüência respiratória e o consumo de oxigênio pelo animal, uma vez que quanto maior o consumo de oxigênio, maior será sua taxa metabólica. Partindo desta premissa, os animais observados neste estudo não apresentaram eficiência fisiológica para controle termorregulatório nas primeiras 24 horas. Entretanto, apesar de não ter sido afetada no período estudado, pode-se observar aumento não significativo de $21 \%$ nos valores médios de frequiência respiratória nos leitões a partir de nove horas de vida. Alguns estudos referenciados por Ferreira (2005), mostraram aumento de 28 a $56 \%$ na taxa metabólica durante as primeiras 48 horas de vida dos leitões.

A temperatura retal dos leitões recém-nascidos aumentou $(\mathrm{P}<0,05)$ após nove horas de nascimento dos leitões. Este aumento da temperatura interna dos animais corrobora a hipótese de que houve tentativa dos animais em manter sua homeotermia logo nas primeiras horas de vida, evidenciando o aumento do metabolismo neste periodo. A disponibilidade energética proveniente da ingestão de colostro logo após o nascimento pode ter contribuído para tal resultado, uma vez que ocorre absorção de lipídeos de cadeia curta provenientes do leite materno, sem necessidade de formação de micelas no intestino proximal em razão da alta eficiência da lípase gástrica neste momento da vida do leitão (LEWIS \& SOUTHERNL, 2001).

Apesar de terem aumentado sua temperatura interna, os leitões recém-nascidos ainda possuem pequena capacidade de regular sua temperatura corporal, principalmente por causa do seu incompleto desenvolvimento hipotalâmico, sendo imprescindível o uso de fontes suplementares de calor nesta fase. Esta recomendação de manejo ficou evidente pela elevação $(\mathrm{P}<0,05)$ observada nas temperaturas superficiais da nuca e da paleta, evidenciando a deficiência de manutenção da homeotermia nas primeiras horas de vida dos leitões. 
Tabela 1 - Resultado dos parâmetros fisiológicos nas primeiras 24 horas de vida dos leitões.

\begin{tabular}{|c|c|c|c|c|c|}
\hline \multirow{2}{*}{ Hora } & \multirow{2}{*}{$\mathrm{FR}(\mathrm{mov} / \mathrm{min})^{1}$} & \multirow{2}{*}{$\begin{array}{c}\text { TR } \\
\left({ }^{\circ} \mathrm{C}\right)\end{array}$} & \multicolumn{3}{|c|}{ Temperatura de superfície } \\
\hline & & & Nuca & Paleta & Pernil $^{1}$ \\
\hline 1 & 51 & $37.60 \mathrm{~b}$ & $36.75 \mathrm{a}$ & $36.86 \mathrm{~b}$ & 36.41 \\
\hline 2 & 41 & $37.32 \mathrm{~b}$ & $35.94 \mathrm{~b}$ & $36.19 \mathrm{~b}$ & 35.75 \\
\hline 3 & 50 & $37.39 \mathrm{~b}$ & $36.64 \mathrm{~b}$ & $36.78 \mathrm{~b}$ & 36.35 \\
\hline 4 & 49 & $37.64 \mathrm{~b}$ & $36.30 \mathrm{~b}$ & $36.90 \mathrm{~b}$ & 34.80 \\
\hline 5 & 42 & $37.36 \mathrm{~b}$ & $36.40 \mathrm{~b}$ & $36.69 \mathrm{~b}$ & 36.09 \\
\hline 6 & 46 & $37.31 \mathrm{~b}$ & $36.30 \mathrm{~b}$ & $36.90 \mathrm{~b}$ & 36.26 \\
\hline 7 & 50 & $37.60 \mathrm{~b}$ & $36.33 \mathrm{~b}$ & $36.33 \mathrm{~b}$ & 35.81 \\
\hline 8 & 49 & $37.46 \mathrm{~b}$ & $36.41 \mathrm{~b}$ & $36.94 \mathrm{~b}$ & 36.13 \\
\hline 9 & 47 & $37.87 \mathrm{a}$ & $37.13 \mathrm{a}$ & $37.36 \mathrm{a}$ & 37.00 \\
\hline 10 & 49 & $37.93 \mathrm{a}$ & $36.91 \mathrm{a}$ & $37.13 \mathrm{a}$ & 36.88 \\
\hline 11 & 51 & $38.02 \mathrm{a}$ & $36.94 \mathrm{a}$ & $36.86 \mathrm{~b}$ & 36.75 \\
\hline 12 & 53 & $37.86 \mathrm{a}$ & $37.00 \mathrm{a}$ & $37.27 \mathrm{a}$ & 36.61 \\
\hline 13 & 63 & $38.04 \mathrm{a}$ & $37.20 \mathrm{a}$ & $37.30 \mathrm{a}$ & 36.93 \\
\hline 14 & 47 & $37.95 \mathrm{a}$ & $37.46 \mathrm{a}$ & $37.25 \mathrm{a}$ & 36.64 \\
\hline 15 & 51 & $38.20 \mathrm{a}$ & $36.86 \mathrm{a}$ & $37.03 \mathrm{~b}$ & 36.50 \\
\hline 16 & 47 & $38.14 \mathrm{a}$ & $36.90 \mathrm{a}$ & $37.06 \mathrm{~b}$ & 36.50 \\
\hline 17 & 48 & $38.09 \mathrm{a}$ & $37.23 \mathrm{a}$ & $37.36 \mathrm{a}$ & 36.96 \\
\hline 18 & 57 & $38.48 \mathrm{a}$ & $36.93 \mathrm{a}$ & $37.36 \mathrm{a}$ & 36.63 \\
\hline 19 & 46 & $38.56 \mathrm{a}$ & $37.06 \mathrm{a}$ & $37.36 \mathrm{a}$ & 36.76 \\
\hline 20 & 48 & $37.88 \mathrm{a}$ & $37.43 \mathrm{a}$ & $37.63 \mathrm{a}$ & 37.16 \\
\hline 21 & 50 & $38.16 \mathrm{a}$ & $37.16 \mathrm{a}$ & $37.54 \mathrm{a}$ & 36.58 \\
\hline 22 & 56 & $38.45 \mathrm{a}$ & $37.53 \mathrm{a}$ & $37.80 \mathrm{a}$ & 37.23 \\
\hline 23 & 51 & $38.14 \mathrm{a}$ & $36.93 \mathrm{a}$ & $37.36 \mathrm{a}$ & 37.03 \\
\hline 24 & 55 & $38.00 \mathrm{a}$ & $36.96 \mathrm{a}$ & $37.03 \mathrm{~b}$ & 36.66 \\
\hline CV\% & 35,29 & 1,96 & 2,58 & 2,53 & 4,78 \\
\hline
\end{tabular}

${ }^{1}$ Não diferem entre si pelo teste $\mathrm{F}(\mathrm{P}>0,05)$.

Médias seguidas pela mesma letra não diferem entre si pelo teste de Scott-Knott à nível de 5\% de probabilidade

Means following for the same letter don't differ amongst themselves for the test of Scott-Knott to at level of 5\% of probability.

As temperaturas superficiais da nuca da paleta, aumentaram $(\mathrm{P}<0,05)$ com o passar das primeiras horas de vida, reforçando o resultado observado de temperatura retal, indicando o aumento da temperatura superficial do animal a partir da nona hora de vida dos leitões. Este fato deve-se ao aumento do metabolismo do animal com o passar das horas, pois o leitão passa de um estado de relação uterina com a mãe, para um estado fisiológico independente .

A temperatura superficial do pernil não foi influenciada $(\mathrm{P}>0,05)$ pelo passar das horas de observação, divergindo dos resultados da temperatura superficial na nuca e paleta. O maior coeficiente de variação obtido nesta variável, pode ter colaborado neste resultado.

Na Figura 1 estão apresentados os resultados do comportamento dos leitões nas primeiras 24 horas de vida.

Os resultados obtidos do estudo comportamental dos leitões demonstraram que eles passaram mais da metade $(53,8 \%)$ do tempo de seu primeiro dia de vida dormindo e somente $31,2 \%$ mamando. Isto se deve, provavelmente, ao metabolismo menos acelerado que estes animais apresentam, em relação ao período subseqüente ainda na lactação. 

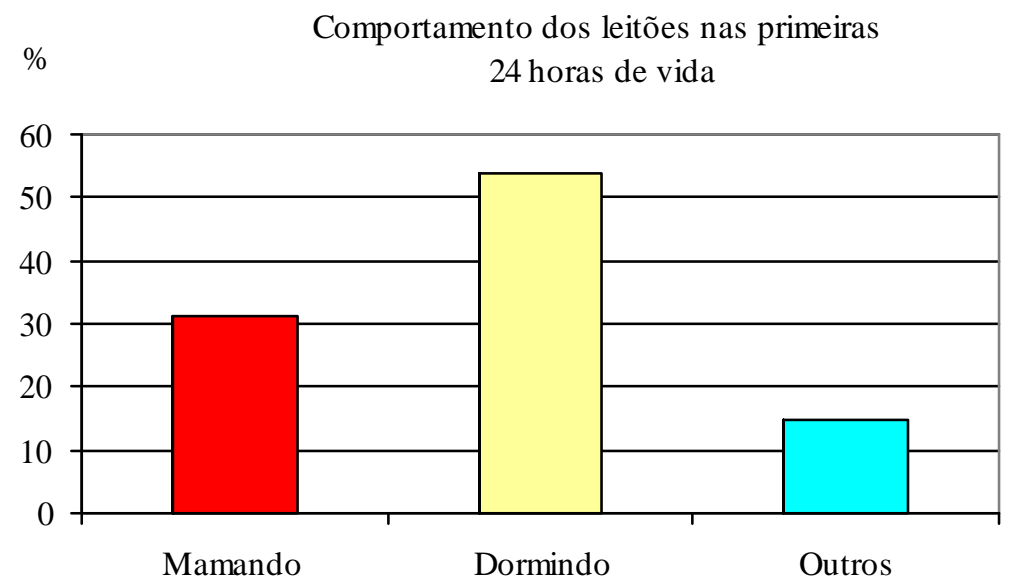

Figura 1 - Comportamento dos leitões nas primeiras 24 horas de vida.

Este resultado indica a necessidade e a importância de se utilizar protetores laterais contra esmagamento acidental de leitões pela matriz, pois a leitegada passa grande parte de suas primeiras horas de vida dormindo, com menor chance de se proteger do deslocamento da matriz quando alojada em baias sem uso de celas parideiras.

\section{CONCLUSÕES}

Leitões geneticamente selecionados para crescimento rápido apresentaram aumento de temperatura retal e superficial como reação à dissipação de calor para o ambiente, evidenciando tentativa de controle termorregulatório a partir da nona hora de vida.

O comportamento dos animais neste período demanda cuidados intensificados de manejo, de modo a reduzir a mortalidade na maternidade.

\section{REFERÊNCIAS BIBLIOGRÁFICAS}

BROWN-BRANDL, T. M.; EIGENBERG, R. A.; NIENABER, J. A. Thermorregulatory profile of a newer genetic line of pigs. Livestock Production Science, Amsterdam, v. 71, p. 253-260, 2001.

DEL-CLARO, K. Comportamento animal: uma introdução à ecologia comportamental. Jundiaí: Conceito, 2004. 132 p.

ENGLISH, P. R. Improving piglet survival, growth to weaning and post weaning performance. In: SEMINÁRIO INTERNACIONAL DE SUINOCULTURA, 3., 1998, São Paulo. Anais... São Paulo: [s.n.], 1998. p. 17-36.
FERREIRA, D. F. Sistema de análises estatísticas SISVAR. Lavras: UFLA, 2000.

FERREIRA, R. A. Maior produção com melhor ambiente para aves, suínos e bovinos. Viçosa: Aprenda Fácil , 2005. $374 \mathrm{p}$.

LEWIS, A. J.; SOUTHERNL, L. Swine nutrition: feeding and feed. 2. ed. Flórida: CRC, 2001. 1009 p.

LIMA, J. A. F. Suinocultura. Lavras: UFLA, 1995. 217 p. Apostila.

PANDORFI, H. Avaliação do comportamento de leitões em diferentes sistemas de aquecimento por meio da análise da imagem e identificação eletrônica. 2002. 89 f. Dissertação (Mestrado em Agronomia - Física do Ambiente Agrícola) - Escola Superior de Agricultura Luiz de Queiroz, Piracicaba, 2002.

PEREIRA, C. Z.; PASSOS, A. A. Informe técnico: cuidados especiais. Revista Suinocultura Industrial, [S.1.], n. 134, set. 1998.

SCOTT, K.; CHENNELLS, D. J.; CAMPBELL, F. N. The welfare of finishing pigs in two contrasting housing systems: fully-slatted versus straw-bedded accommodation. Livestock Production Science, Amsterdam, v. 103, p. 104-115, 2006. 